\title{
Research Paper: The Effect of Knee Osteoarthritis on Excursions of Lower Limb Joints During Gait
}

\author{
Ali Jalalvand ${ }^{1 *}$, Mehrdad Anbarian², Shahram Ahanjan ${ }^{3}$, Behrouz Hajiloo², Ali Akbar Tayfe Seyedan ${ }^{1}$ \\ 1. Department of Physical Education, Faculty of Human Sciences, Hamedan Branch, Islamic Azad University, Hamedan, Iran. \\ 2. Department of Sport Biomechanics, Faculty of Sport Sciences, Bu-Ali Sina University, Hamedan, Iran. \\ 3. Department of Physical Education, Amirkabir University of Technology, Tehran, Iran.
}

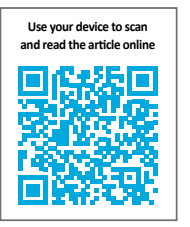

Citation: Jalalvand Ali, Anbarian M, Ahanjan Sh, Hajiloo B, Tayfe Seyedan AA. The Effect of Knee Osteoarthritis on Excursions of Lower Limb Joints During Gait. Physical Treatments. 2017; 6(4):233-241. https://doi.org/10.18869/NRIP.PTJ.6.4.233

: https://doi.org/10.18869/NRIP.PTJ.6.4.233

Article info:

Received: 16 May 2016

Accepted: 30 Sep. 2016
Keywords:

Severity of osteoarthritis, Joint excursion, Gait

\begin{abstract}
A B S T R A C T
Purpose: This study aimed to investigate excursion changes at the hip, knee, and ankle joints during gait in patients with knee osteoarthritis (KOA) of varying severity.

Methods: The method of research was causal-comparative (Ex Post Facto). A Vicon motion analysis system with $4 \mathrm{~T}$-Series cameras was used to measure the kinematics variables. Fifteen normal subjects without KOA and thirty patients with KOA participated in this study. According to Kellgren and Lawrence radiologic scale, the patients were divided into three groups of mild $(\mathrm{n}=10)$, moderate $(\mathrm{n}=10)$, and severe $(\mathrm{n}=10)$. Kinematics parameters of gait includes the range of motion (ROM) in all three planes of motion at the hip, knee, and ankle joints during gait, which were calculated by Vicon Nexus 1.8.5 software. All data were extracted by Polygon 3.5.1 and then were analyzed in SPSS20 using 1-way ANOVA test. The level of significance was set at $\mathrm{P}<0.05$.

Results: There were no significant differences in all kinematics parameters (joint angles) between the healthy and KOA groups $(\mathrm{P}>0.05)$. There were significant differences observed between groups of mild, moderate, and severe KOA with healthy one with respect to foot progress angles in the transverse plane, foot eversion/inversion excursion, knee flexion/extension excursion, knee abduction/adduction excursion, hip internal/external rotation excursion, anterior/posterior pelvic tilt, and lateral pelvic tilt $(\mathrm{P}<0.05)$.

Conclusion: The results showed that some ROM parameters in all three planes of motion can estimate the severity of osteoarthritis. Some observed changes in kinematics parameters are due to effects of compensatory mechanisms, and some might be considered as the severity of the osteoarthritis. In addition, osteoarthritis in one joint had strong effects on other joints.
\end{abstract}

\section{Introduction}

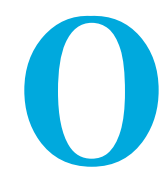

steoarthritis $(\mathrm{OA})$ is the most common musculoskeletal complaint. Characteristics of OA include articular cartilage de- generation, narrowing of joint space, pain, and lack of ability. Knee osteoarthritis is a multifactorial disease that can affect many joints. The symptoms of OA such as the emergence of pain and stiffness of the joint and muscle weakness are strong risk factors for mobility limitation

\section{* Corresponding Author:}

Ali Jalalvand, PhD

Address: Department of Physical Education, Faculty of Human Sciences, Hamedan Branch, Islamic Azad University, Hamedan, Iran.

Phone: +98 (918) 9513172

E-mail:jalalvand_ali@yahoo.com 
and impaired quality of life $[1,2]$. Signs and indications of OA include stiffness, loss of flexibility, activity limitations, fatigue, as well as swollen and painful joint. OA is a very common health problem worldwide affecting performance at work and general well-being, and bears huge economical costs [3].

Gait adaptation occurs soon after walking in response to pain, deformity, or laxity in the lower limb joints. It is primarily related to disease progression rate; antalgic gait is often adapted the patients with OA progression. Gait adaptation depends on the severity of OA, pain, muscle weakness, or limited range of passive motions. In addition, these adaptations may be affected by other lower extremity joints [4].

Fewer studies report the role played by other lower extremity joints in facilitating compensatory mechanics of the knee in patients with OA during walking. Stauffer et al. found that patients with knee OA had significantly less dynamic knee flexion (range of motion) during gait [5]. In six research studies on osteoarthritis, individuals with knee osteoarthritis had less knee flexion compared to healthy subjects [6]. Kaufman et al. reported that the female subjects had significantly greater knee flexion and knee extensor moment, suggesting a mechanism for reducing articular forces of the painful and unstable knee joint [7]. Thus, such studies could be valuable tools for better evaluation of compensatory mechanics in people with osteoarthritis of the knee. In addition, this viewpoint may be useful to understand how motor locomotor improves in therapeutic intervention [8]. In this regard, the main objective of our study was to see if there were changes in kinematics of the lower extremity joints and or compensation strategies at different stages of disease during walking.

Most gait studies have characterized differences between subjects classified as either knee osteoarthritis or asymptomatic [9]. As a degenerative disease, knee OA might well be caused by biochemical or mechanical problems. In order to enrich our understanding of biomechanical changes of OA, gait analysis has been conducted. Some studies have targeted single population of patients with various or specified stages of OA and compared the results with the states of asymptomatic subjects as control. The degenerative alterations in the knee joint, however, often develop based on the progression of OA. Also of importance is the fact that OA patients may have changes in muscle strength, range of motion, and alignment related to disability associated with this disease. They may also have different kinds of kinematics based on their OA stages. It is somewhat difficult to find about the stage-specific biomechanical changes of the OA from a single study community [10]

Although the alterations of kinematics in the sagittal plane of knee OA patients is almost clear, very little is known about the frontal and horizontal planes. Research has shown that OA may cause temporo-spatial gait parameters and kinematic and kinetic alterations in the sagittal plane of motion during gait [7, 9, 11-13]. However, little research has been done on the effect of the radiographic severity of knee OA on these parameters $[9,14]$. Fewer still have investigated the effects of knee OA severity on the biomechanics of other joints in the lower limbs. A specific aspect of advanced osteoarthritis gait has been detected and investigated in such studies.

Based on results from these studies, we might well assume that the detected locomotor perturbation caused by the compensatory or adaptive mechanism is also due to severe OA. This fact must definitely be considered when comparing different study groups with different levels of OA of the biomechanics of other joints located in the lower limbs and also in the frontal and horizontal planes. $\mathrm{OA}$ is most commonly found in one joint, especially in its specific stages, however, it is well-documented that malfunction of one joint would definitely result in the overall disability and changes in the functions of other joints. The knee joint linked to adjacent joints and affect them. In other words, if a single joint does not work poperly, the joints above and below it can be affected. Limited studies have examined the impact of osteoarthritis of the knee on the ankle and hip joints [15].

Furthermore, more studies of knee OA have focused on late-stage of the disease. And a little research has conducted on the biomechanical effects in other disease stages and so little biomechanical information is available for the different stages of the disease. Such studies can help slow the development of these diseases and pain relief measures [16].

\section{Materials and Methods}

In this causal-comparative study (Ex Post Facto), 15 healthy males without knee $\mathrm{OA}$ and 30 male patient with bilateral knee OA were recruited from the Orthopedic and Medicine Clinic of Hamedan City. Sampling was purposive. The sample size is determined based on previous similar studies [17]. Patient's OA was diagnosed through history, inspection, and radiologic assessments. Inclusion criteria were having radiological signs of $\mathrm{OA}$; 
no history of orthopedic knee injuries such as meniscus and ligament injuries, neurological or cardiopulmonary diseases, which might affect the patient's gait; history of chronic knee pain for the last 6 months or longer; no history of previous lower extremity surgery, major trauma, tendinopathy, bursitis, serious hearing, sight or speech defects; and no osteoarthritis diseases, gout, rheumatoid arthritis involving other joints in the lower extremities.

The study patients were divided according to the Kellgren and Lawrence radiologic scale, WOMAC index, and visual analog scale in three groups of mild $(n=10)$, moderate $(n=10)$, and severe $(n=10)$ knee OA. Also the normal subjects were 15 individuals without knee OA. Subjects walked with a self-selected speed along the walking path to do five successful trials. Ethical approval was received from Ethics Committee of Hamedan University of Medical Sciences, Hamedan. Written informed consent was obtained from each subject before data collection.

Vicon motion analysis system with 4 cameras $(130 \mathrm{~Hz})$ was used to record the motions of the segments and reaction forces during gait assessment. A low pass filter (Butterworth) was used to filter the kinematic data with a cut off frequency of $10 \mathrm{~Hz}$. Measurement errors of the kinematic evaluations was about $0.2 \mathrm{~mm}$ using Nexus software. Cameras were placed on both sides of the walkway at a distance of $5 \mathrm{~m}$ from the center of the calibrated space. The walkway path along the laboratory was 18 $\mathrm{m}$. The calibration space dimensions were $300 \times 150 \times 200$ $\mathrm{mm}$ (length, width, and height, respectively). The participants walked $7 \mathrm{~m}$ before entering the calibrated space. The calibration frame length allowed full left and right strides to be placed in the calibrated frame.

Sixteen $25 \mathrm{~mm}$ diameter markers were placed on left and right superior anterior iliac spine, superior posterior iliac spine, thigh, lateral condyle of the knee, shank, lateral malleoli, heel, and second distal metatarsal. The markers reflected the infrared light toward the cameras. All markers were placed according to Plug-in-Gait Protocol
[18]. After calibrating the cameras, the anthropometric data, including weight, height, leg length, knee and ankle width, and the distance of left and right anterior superior iliac spine were recorded in the Nexus software. These data are necessary for kinetics and kinematics analyses.

The subjects were asked to walk with a comfortable speed to accomplish 5 successful trials. The mean values of 5 trials were used for each parameter. Data were calculated by Vicon Nexus 1.8.5 software and were extracted by Polygon 3.5.1. The normal distribution of the data was evaluated using Shapiro-Wilk test. The assumption of homogeneity of variance was tested using Levene's test of equality of variances $(\mathrm{P}>0.05)$. Data were analyzed by SPSS20 doing 1-way analysis of variance (ANOVA) test following Bonferroni post hoc test. The level of significance was set at $\mathrm{P}<0.05$.

\section{Results}

Table 1 presents the demographic characteristics of the four groups. No difference was observed between the groups with respect to their age, height, or weight (Table 1). The descriptive characteristics of excursions of lower limb joints during gait in participants are shown in Table 2. The difference of means of the joint excursions at the hip, knee, and ankle joints of normal and the patients with knee OA are shown in Table 3. As seen in Table 3, there were no significant differences in the dominant and non-dominant extremity between groups in the range of ankle plantar flexion/dorsiflexion, ankle internal/external rotation, hip flexion/extension, hip abduction/adduction, internal/external hip rotation $(\mathrm{P}>0.05)$.

There were significant difference in foot progress angles in the transverse plane in the non-dominant extremity between groups of mild $\mathrm{OA}$ and moderate $\mathrm{OA}$ $(\mathrm{P}=0.047)$, foot eversion/inversion excursion in the nondominant extremity between groups of normal and mild $\mathrm{OA}(\mathrm{P}=0.010)$, the non-dominant extremity between groups of mild OA and severe $\mathrm{OA}(\mathrm{P}=0.005)$.

Table 1. Demographic characteristics of patients and healthy subjects (Mean \pm SD)

\begin{tabular}{|c|c|c|c|c|c|}
\hline \multirow{2}{*}{ Variables } & \multicolumn{4}{|c|}{ Groups } & \multirow{2}{*}{$\mathbf{P}$} \\
\hline & Mild & Moderate & Severe & Normal & \\
\hline Age (y) & $59.16 \pm 8.28$ & $54.33 \pm 12.06$ & $62.60 \pm 8.53$ & $48.80 \pm 6.61$ & 0.129 \\
\hline Height $(\mathrm{cm})$ & $1.70 \pm 5.60$ & $1.68 \pm 7.11$ & $1.67 \pm 6.46$ & $1.67 \pm 6.76$ & 0.914 \\
\hline Weight (kg) & $76.16 \pm 8.47$ & $72 \pm 9.50$ & $81.80 \pm 13$ & $76.60 \pm 11.80$ & 0.526 \\
\hline
\end{tabular}


Table 2. Descriptive characteristics of the osteoarthritis subjects and healthy group (Mean \pm SD) over a complete gait cycle

\begin{tabular}{|c|c|c|c|c|c|}
\hline \multirow{2}{*}{ Variables } & \multirow{2}{*}{ Lower Extremity } & \multicolumn{4}{|c|}{ Groups } \\
\hline & & Normal & Mild Osteoarthritis & Moderate Osteoarthritis & Severe Osteoarthritis \\
\hline \multirow{3}{*}{ Foot progress angles } & Dominant & $17.83 \pm 8.82$ & $11.11 \pm 2.66$ & $15.04 \pm 3.93$ & $13.06 \pm 4.75$ \\
\hline & & & & & \\
\hline & Non-dominant & $14.19 \pm 2.64$ & $16.53 \pm 5.38$ & $11.72 \pm 3.53$ & $12.92 \pm 3.13$ \\
\hline \multirow{2}{*}{$\begin{array}{l}\text { Ankle plantar flexion/dor- } \\
\text { siflexion }\end{array}$} & Dominant & $27.52 \pm 5.68$ & $25.91 \pm 3.55$ & $25.98 \pm 5.85$ & $25.70 \pm 5.08$ \\
\hline & Non-dominant & $28.71 \pm 6.01$ & $30.0 \pm 5.90$ & $26.46 \pm 3.35$ & $26.14 \pm 11.7$ \\
\hline \multirow{2}{*}{$\begin{array}{l}\text { Subtalar inversion/ever- } \\
\text { sion }\end{array}$} & Dominant & $4.25 \pm 3.89$ & $6.18 \pm 3.31$ & $3.49 \pm 2.79$ & $5.09 \pm 1.75$ \\
\hline & Non-dominant & $3.97 \pm 1.24$ & $7.04 \pm 2.20$ & $5.56 \pm 2.07$ & $3.63 \pm 0.908$ \\
\hline \multirow{2}{*}{$\begin{array}{l}\text { Ankle internal/external } \\
\text { rotation }\end{array}$} & Dominant & $32.89 \pm 13.47$ & $35.80 \pm 11.76$ & $26.96 \pm 9.91$ & $24.39 \pm 7.69$ \\
\hline & Non-dominant & $36.31 \pm 18.94$ & $33.46 \pm 11.94$ & $27.98 \pm 4.30$ & $21.85 \pm 6.61$ \\
\hline \multirow{2}{*}{ Knee flexion/extension } & Dominant & $61.19 \pm 8.69$ & $54.95 \pm 9.55$ & $54.12 \pm 2.87$ & $43.34 \pm 9.68$ \\
\hline & Non-dominant & $61.80 \pm 8.65$ & $58.08 \pm 8.61$ & $57.30 \pm 3.01$ & $46.32 \pm 14.53$ \\
\hline \multirow{2}{*}{ Knee abduction/adduction } & Dominant & $15.43 \pm 9.51$ & $17.57 \pm 4.98$ & $21.61 \pm 7.09$ & $28.83 \pm 11.86$ \\
\hline & Non-dominant & $22.65 \pm 7.59$ & $20.33 \pm 8.83$ & $19.19 \pm 10.64$ & $15.75 \pm 7.74$ \\
\hline \multirow{2}{*}{$\begin{array}{l}\text { Knee internal/external } \\
\text { rotation }\end{array}$} & Dominant & $30.19 \pm 17.63$ & $32.21 \pm 17.27$ & $19.84 \pm 8.23$ & $16.18 \pm 4.52$ \\
\hline & Non-dominant & $25.33 \pm 12.79$ & $31.28 \pm 10.93$ & $21.30 \pm 6.14$ & $15.49 \pm 10.02$ \\
\hline \multirow{3}{*}{ Hip flexion/extension } & Dominant & $44.29 \pm 5.10$ & $37.94 \pm 5.76$ & $40.24 \pm 6.27$ & $41.89 \pm 6.40$ \\
\hline & & & & & \\
\hline & Non-dominant & $45.46 \pm 6.13$ & $41.54 \pm 5.19$ & $43.39 \pm 4.53$ & $40.59 \pm 13.71$ \\
\hline \multirow{3}{*}{ Hip abduction/adduction } & Dominant & $12.13 \pm 2.83$ & $9.08 \pm 2.47$ & $9.95 \pm 4.38$ & $10.63 \pm 2.91$ \\
\hline & & & & & \\
\hline & Non-dominant & $13.54 \pm 1.00$ & $12.13 \pm 2.50$ & $11.68 \pm 2.72$ & $10.32 \pm 4.04$ \\
\hline \multirow{2}{*}{$\begin{array}{l}\text { Hip internal/external } \\
\text { rotation }\end{array}$} & Dominant & $15.65 \pm 3.57$ & $22.31 \pm 4.94$ & $27.49 \pm 6.77$ & $31.61 \pm 5.31$ \\
\hline & Non-dominant & $16.71 \pm 3.76$ & $20.49 \pm 7.70$ & $27.43 \pm 10.15$ & $26.31 \pm 4.00$ \\
\hline \multirow{2}{*}{$\begin{array}{c}\text { Anterior/posterior pelvic } \\
\text { tilt }\end{array}$} & Dominant & $5.09 \pm 2.26$ & $3.93 \pm 3.19$ & $5.33 \pm 2.48$ & $8.71 \pm 2.03$ \\
\hline & Non-dominant & $5.22 \pm 2.69$ & $3.98 \pm 1.81$ & $5.25 \pm .78$ & $8.99 \pm 5.70$ \\
\hline \multirow{2}{*}{ Lateral pelvic tilt } & Dominant & $6.87 \pm 2.75$ & $4.21 \pm 1.92$ & $5.85 \pm 1.83$ & $5.31 \pm 2.07$ \\
\hline & Non-dominant & $7.17 \pm 1.99$ & $4.43 \pm 1.44$ & $6.74 \pm 2.21$ & $4.14 \pm 0.548$ \\
\hline \multirow{3}{*}{ Pelvic rotation } & Dominant & $8.72 \pm 3.22$ & $8.07 \pm 3.16$ & $9.15 \pm 4.22$ & $8.94 \pm 1.93$ \\
\hline & & & & & \\
\hline & Non-dominant & $9.54 \pm 1.56$ & $9.47 \pm 3.61$ & $9.95 \pm 4.70$ & $6.88 \pm 3.39$ \\
\hline
\end{tabular}

As seen in Table 3 , there were statistically significant differences between the dominant $(\mathrm{P}=0.003)$ and nondominant extremity $(\mathrm{P}=0.017)$ in the range of knee flexion/extension excursion of the normal group compared to the severe OA group, the dominant $(\mathrm{P}=0.029)$ and nondominant extremity $(\mathrm{P}=0.05)$ in the range of knee flexion/ extension excursion of the mild group compared to the severe OA group, the dominant extremity between groups of moderate $\mathrm{OA}$ compared to severe $\mathrm{OA}(\mathrm{P}=0.041)$, the dominant extremity $(\mathrm{P}=0.023)$ in the range of knee abduction/adduction excursion of the normal group compared to the severe OA group, in the dominant extremity between groups of mild OA compared to severe OA $(\mathrm{P}=0.042)$, and the non-dominant extremity $(\mathrm{P}=0.019)$ in 
Table 3. Comparing range of motion between dominant and non-dominant extremities in all three planes of motion at the hip, knee, and ankle joints during gait

\begin{tabular}{|c|c|c|c|c|c|c|c|}
\hline Variables & & $\begin{array}{l}\text { Normal vs. } \\
\text { Mild OA }\end{array}$ & $\begin{array}{l}\text { Normal vs. } \\
\text { Moderate OA }\end{array}$ & $\begin{array}{l}\text { Normal vs. } \\
\text { Severe OA }\end{array}$ & $\begin{array}{l}\text { Mild OA vs. } \\
\text { Moderate OA }\end{array}$ & $\begin{array}{l}\text { Mild OA vs. } \\
\text { Severe OA }\end{array}$ & $\begin{array}{c}\text { Moderate } \\
\text { vs. Severe } \\
\text { OA }\end{array}$ \\
\hline \multirow{2}{*}{ Foot progress angles } & Dominant & $\begin{array}{l}M d=6.72 \\
P=0.053\end{array}$ & $\begin{array}{l}M d=2.79 \\
P=0.400\end{array}$ & $\begin{array}{l}M d=4.77 \\
P=0.175\end{array}$ & $\begin{array}{l}M d=3.92 \\
P=0.220\end{array}$ & $\begin{array}{l}M d=1.94 \\
P=0.555\end{array}$ & $\begin{array}{l}M d=1.98 \\
P=0.549\end{array}$ \\
\hline & Non-dominant & $\begin{array}{l}M d=2.34 \\
P=0.335\end{array}$ & $\begin{array}{l}M d=2.47 \\
P=0.310\end{array}$ & $\begin{array}{l}M d=1.26 \\
P=0.615\end{array}$ & $\begin{array}{l}\mathrm{Md}=4.81 \\
\mathrm{P}=0.047^{*}\end{array}$ & $\begin{array}{l}M d=3.60 \\
P=0.145\end{array}$ & $\begin{array}{l}M d=1.20 \\
P=0.616\end{array}$ \\
\hline \multirow{2}{*}{$\begin{array}{c}\text { Ankle plantar flexion/ } \\
\text { dorsiflexion }\end{array}$} & Dominant & $\begin{array}{l}M d=1.61 \\
P=0.608\end{array}$ & $\begin{array}{l}M d=1.53 \\
P=0.624\end{array}$ & $\begin{array}{l}\mathrm{Md}=1.82 \\
\mathrm{P}=0.578\end{array}$ & $\begin{aligned} \mathrm{Md} & =0.0718 \\
\mathrm{P} & =0.981\end{aligned}$ & $\begin{array}{c}M d=0.216 \\
P=0.945\end{array}$ & $\begin{array}{c}M d=0.287 \\
P=0.927\end{array}$ \\
\hline & Non-dominant & $\begin{array}{l}M d=1.29 \\
P=0.769\end{array}$ & $\begin{array}{l}M d=2.25 \\
P=0.611\end{array}$ & $\begin{array}{l}M d=2.57 \\
P=0.611\end{array}$ & $\begin{array}{l}M d=3.54 \\
P=0.404\end{array}$ & $\begin{array}{l}M d=3.86 \\
P=0.386\end{array}$ & $\begin{array}{c}M d=0.320 \\
P=0.942\end{array}$ \\
\hline \multirow{2}{*}{$\begin{array}{c}\text { Subtalar inversion/ } \\
\text { eversion }\end{array}$} & Dominant & $\begin{array}{l}M d=1.92 \\
P=0.309\end{array}$ & $\begin{array}{c}M d=0.758 \\
P=0.686\end{array}$ & $\begin{array}{l}M d=0.846 \\
P=0.665\end{array}$ & $\begin{array}{l}M d=2.68 \\
P=0.144\end{array}$ & $\begin{array}{c}M d=1.083 \\
P=0.564\end{array}$ & $\begin{array}{l}M d=1.60 \\
P=0.395\end{array}$ \\
\hline & Non-dominant & $\begin{array}{l}M d=3.07 \\
P=0.010^{* *}\end{array}$ & $\begin{array}{l}M d=1.59 \\
P=0.151\end{array}$ & $\begin{array}{l}M d=0.339 \\
P=0.763\end{array}$ & $\begin{array}{l}M d=1.48 \\
P=0.160\end{array}$ & $\begin{array}{l}\mathrm{Md}=3.41 \\
\mathrm{P}=0.005^{* *}\end{array}$ & $\begin{array}{l}M d=1.93 \\
P=0.086\end{array}$ \\
\hline \multirow{2}{*}{$\begin{array}{l}\text { Ankle internal/external } \\
\text { rotation }\end{array}$} & Dominant & $\begin{array}{l}M d=2.90 \\
P=0.666\end{array}$ & $\begin{array}{l}M d=5.93 \\
P=0.382\end{array}$ & $\begin{array}{l}M d=8.50 \\
P=0.234\end{array}$ & $\begin{array}{l}\mathrm{Md}=8.83 \\
\mathrm{P}=0.178\end{array}$ & $\begin{array}{c}M d=11.40 \\
P=0.102\end{array}$ & $\begin{array}{l}M d=2.56 \\
P=0.702\end{array}$ \\
\hline & Non-dominant & $\begin{array}{c}M d=2.85 \\
P=0.689\end{array}$ & $\begin{array}{c}M d=8.32 \\
P=0.251\end{array}$ & $\begin{array}{c}\mathrm{Md}=14.45 \\
\mathrm{P}=0.064\end{array}$ & $\begin{array}{c}M d=5.47 \\
P=0.424\end{array}$ & $\begin{array}{c}M d=11.60 \\
P=0.115\end{array}$ & $\begin{array}{c}M d=6.13 \\
P=0.394\end{array}$ \\
\hline \multirow{2}{*}{ Knee flexion/extension } & Dominant & $\begin{array}{l}M d=6.24 \\
P=0.218\end{array}$ & $\begin{array}{l}M d=7.07 \\
P=0.165\end{array}$ & $\begin{array}{l}M d=17.85 \\
P=0.003^{* *}\end{array}$ & $\begin{array}{c}M d=0.827 \\
P=0.861\end{array}$ & $\begin{array}{l}M d=11.60 \\
P=0.029^{*}\end{array}$ & $\begin{array}{c}\mathrm{Md}=10.78 \\
\mathrm{P}=0.041^{*}\end{array}$ \\
\hline & Non-dominant & $\begin{array}{l}M d=3.71 \\
P=0.519\end{array}$ & $\begin{array}{l}M d=4.49 \\
P=0.435\end{array}$ & $\begin{array}{l}M d=15.47 \\
P=0.017^{*}\end{array}$ & $\begin{array}{c}M d=0.785 \\
P=0.885\end{array}$ & $\begin{array}{c}M d=11.75 \\
P=0.05^{*}\end{array}$ & $\begin{array}{c}M d=10.97 \\
P=0.068\end{array}$ \\
\hline \multirow{2}{*}{$\begin{array}{l}\text { Knee abduction/ad- } \\
\text { duction }\end{array}$} & Dominant & $\begin{array}{l}M d=2.13 \\
P=0.683\end{array}$ & $\begin{array}{l}M d=6.17 \\
P=0.246\end{array}$ & $\begin{array}{l}M d=13.39 \\
P=0.023^{*}\end{array}$ & $\begin{array}{l}M d=4.03 \\
P=0.422\end{array}$ & $\begin{array}{l}M d=11.25 \\
P=0.042^{*}\end{array}$ & $\begin{array}{l}M d=7.22 \\
P=0.178\end{array}$ \\
\hline & Non-dominant & $\begin{array}{l}M d=2.31 \\
P=0.672\end{array}$ & $\begin{array}{l}M d=3.45 \\
P=0.530\end{array}$ & $\begin{array}{l}M d=6.89 \\
P=0.236\end{array}$ & $\begin{array}{l}M d=1.13 \\
P=0.827\end{array}$ & $\begin{array}{l}M d=4.57 \\
P=0.407\end{array}$ & $\begin{array}{l}M d=3.44 \\
P=0.531\end{array}$ \\
\hline \multirow{2}{*}{$\begin{array}{l}\text { Knee internal/external } \\
\text { rotation }\end{array}$} & Dominant & $\begin{array}{l}M d=2.02 \\
P=0.804\end{array}$ & $\begin{array}{c}M d=10.34 \\
P=0.213\end{array}$ & $\begin{array}{l}M d=14 \\
P=0.112\end{array}$ & $\begin{array}{c}M d=12.36 \\
P=0.123\end{array}$ & $\begin{array}{c}M d=16.02 \\
P=0.061\end{array}$ & $\begin{array}{l}M d=3.65 \\
P=0.654\end{array}$ \\
\hline & Non-dominant & $\begin{array}{l}M d=5.94 \\
P=0.345\end{array}$ & $\begin{array}{l}M d=4.03 \\
P=0.518\end{array}$ & $\begin{array}{l}M d=9.84 \\
P=0.141\end{array}$ & $\begin{array}{l}M d=9.98 \\
P=0.105\end{array}$ & $\begin{array}{l}\mathrm{Md}=15.79 \\
\mathrm{P}=0.019^{*}\end{array}$ & $\begin{array}{l}M d=5.81 \\
P=0.356\end{array}$ \\
\hline \multirow{2}{*}{ Hip flexion/extension } & Dominant & $\begin{array}{l}M d=6.35 \\
P=0.098\end{array}$ & $\begin{array}{l}M d=4.04 \\
P=0.273\end{array}$ & $\begin{array}{l}M d=2.39 \\
P=0.530\end{array}$ & $\begin{array}{l}M d=2.30 \\
P=0.508\end{array}$ & $\begin{array}{l}M d=3.95 \\
P=0.284\end{array}$ & $\begin{array}{l}M d=1.65 \\
P=0.650\end{array}$ \\
\hline & Non-dominant & $\begin{array}{l}M d=3.91 \\
P=0.427\end{array}$ & $\begin{array}{l}M d=2.06 \\
P=0.673\end{array}$ & $\begin{array}{l}M d=4.86 \\
P=0.347\end{array}$ & $\begin{array}{l}M d=1.84 \\
P=0.693\end{array}$ & $\begin{array}{c}M d=0.946 \\
P=0.847\end{array}$ & $\begin{array}{l}M d=2.79 \\
P=0.570\end{array}$ \\
\hline \multirow{2}{*}{ Hip abduction/adduction } & Dominant & $\begin{array}{l}M d=3.05 \\
P=0.140\end{array}$ & $\begin{array}{l}\mathrm{Md}=2.18 \\
\mathrm{P}=0.284\end{array}$ & $\begin{array}{l}M d=1.50 \\
P=0.477\end{array}$ & $\begin{array}{c}M d=0.869 \\
P=0.651\end{array}$ & $\begin{array}{l}M d=1.55 \\
P=0.443\end{array}$ & $\begin{array}{c}M d=0.685 \\
P=0.733\end{array}$ \\
\hline & Non-dominant & $\begin{array}{l}M d=1.40 \\
P=0.412\end{array}$ & $\begin{array}{l}M d=1.85 \\
P=0.283\end{array}$ & $\begin{array}{l}M d=3.21 \\
P=0.083\end{array}$ & $\begin{array}{l}M d=0.447 \\
P=0.782\end{array}$ & $\begin{array}{l}M d=1.81 \\
P=0.294\end{array}$ & $\begin{array}{l}M d=1.36 \\
P=0.427\end{array}$ \\
\hline \multirow{2}{*}{$\begin{array}{c}\text { Hip Internal/external } \\
\text { rotation }\end{array}$} & Dominant & $\begin{array}{l}M d=6.65 \\
P=0.055\end{array}$ & $\begin{array}{l}M d=11.84 \\
P=0.002^{* *}\end{array}$ & $\begin{array}{l}M d=15.96 \\
P=0.000^{* *}\end{array}$ & $\begin{array}{l}M d=5.18 \\
P=0.111\end{array}$ & $\begin{array}{l}M d=9.30 \\
P=0.010^{* *}\end{array}$ & $\begin{array}{l}M d=4.11 \\
P=0.220\end{array}$ \\
\hline & Non-dominant & $\begin{array}{l}M d=3.78 \\
P=0.397\end{array}$ & $\begin{array}{l}M d=10.72 \\
P=0.024^{*}\end{array}$ & $\begin{array}{l}M d=9.60 \\
P=0.049^{*}\end{array}$ & $\begin{array}{l}M d=6.94 \\
P=0.112\end{array}$ & $\begin{array}{l}M d=5.82 \\
P=0.198\end{array}$ & $\begin{array}{l}M d=1.11 \\
P=0.800\end{array}$ \\
\hline \multirow{2}{*}{$\begin{array}{l}\text { Anterior/posterior } \\
\text { pelvic tilt }\end{array}$} & Dominant & $\begin{array}{l}M d=1.16 \\
P=0.464\end{array}$ & $\begin{array}{c}M d=0.240 \\
P=0.879\end{array}$ & $\begin{array}{l}M d=3.61 \\
P=0.039^{*}\end{array}$ & $\begin{array}{l}M d=1.40 \\
P=0.356\end{array}$ & $\begin{array}{l}\mathrm{Md}=4.78 \\
\mathrm{P}=0.007^{* *}\end{array}$ & $\begin{array}{l}\mathrm{Md}=3.37 \\
\mathrm{P}=0.044^{*}\end{array}$ \\
\hline & Non-dominant & $\begin{array}{l}M d=1.23 \\
P=0.525\end{array}$ & $\begin{array}{c}M d=0.026 \\
P=0.989\end{array}$ & $\begin{array}{l}M d=3.77 \\
P=0.075\end{array}$ & $\begin{array}{l}M d=1.26 \\
P=0.497\end{array}$ & $\begin{array}{c}M d=5 \\
P=0.017^{*}\end{array}$ & $\begin{array}{l}M d=3.74 \\
P=0.065\end{array}$ \\
\hline Lateral nelvic tilt & Dominant & $\begin{array}{l}M d=2.66 \\
P=0.055\end{array}$ & $\begin{array}{l}M d=1.02 \\
P=0.443\end{array}$ & $\begin{array}{l}M d=1.56 \\
P=0.264\end{array}$ & $\begin{array}{l}M d=1.64 \\
P=0.201\end{array}$ & $\begin{array}{l}M d=1.09 \\
P=0.409\end{array}$ & $\begin{array}{c}M d=0.544 \\
P=0.680\end{array}$ \\
\hline Lateral pelvic tilt & Non-dominant & $\begin{array}{l}M d=2.73 \\
P=0.016^{*}\end{array}$ & $\begin{array}{c}M d=0.427 \\
P=0.683\end{array}$ & $\begin{array}{l}M d=3.02 \\
P=0.012^{*}\end{array}$ & $\begin{array}{l}M d=2.30 \\
P=0.030^{*}\end{array}$ & $\begin{array}{c}M d=0.286 \\
P=0.784\end{array}$ & $\begin{array}{l}\mathrm{Md}=2.59 \\
\mathrm{P}=0.021^{*}\end{array}$ \\
\hline Pelvic rotation & Dominant & $\begin{array}{c}M d=0.652 \\
P=0.748\end{array}$ & $\begin{array}{c}M d=0.421 \\
P=0.835\end{array}$ & $\begin{array}{c}M d=0.218 \\
P=0.918\end{array}$ & $\begin{array}{l}M d=1.07 \\
P=0.580\end{array}$ & $\begin{array}{c}M d=0.870 \\
P=0.668\end{array}$ & $\begin{array}{c}M d=0.203 \\
P=0.920\end{array}$ \\
\hline & Non-dominant & $\begin{array}{c}\mathrm{Md}=0.070 \\
\mathrm{P}=0.971\end{array}$ & $\begin{array}{c}M d=0.402 \\
P=0.855\end{array}$ & $\begin{array}{l}M d=2.66 \\
P=0.255\end{array}$ & $\begin{array}{c}M d=0.473 \\
P=0.822\end{array}$ & $\begin{array}{l}M d=2.59 \\
P=0.248\end{array}$ & $\begin{array}{c}M d=3.06 \\
P=0.175\end{array}$ \\
\hline
\end{tabular}

Md: Mean Difference

PHYSICAL TREA T MENTS

$P$ : The level of significance

${ }^{*} \mathrm{P}<0.05 ;{ }^{* *} \mathrm{P}<0.01$ 
the range of knee internal/external rotation excursion of the mild group compared to the severe OA group.

There were statistically significant differences between the dominant $(\mathrm{P}=0.002)$ and non-dominant extremity $(\mathrm{P}=0.024)$ regarding the range of hip internal/external rotation excursion of the normal group compared to the moderate OA group, the dominant extremity $(\mathrm{P}=0.000)$ and non-dominant extremity $(\mathrm{P}=0.049)$ between groups of normal OA compared to severe $\mathrm{OA}$, and the dominant extremity $(\mathrm{P}=0.010)$ between groups of mild OA compared to severe OA.

There were statistically significant differences between the dominant $(\mathrm{P}=0.039)$ in the range of anterior/posterior pelvic tilt excursion of the normal group compared to the severe OA group, in the dominant extremity $(\mathrm{P}=0.007)$ and non-dominant extremity $(\mathrm{P}=0.017)$ between groups of mild OA compared to severe OA, and in the dominant extremity $(\mathrm{P}=0.044)$ between groups of moderate OA compared to severe OA. Table 3 shows statistically significant differences between the non-dominant $(\mathrm{P}=0.003)$ in the range of lateral pelvic tilt excursion of the normal group compared to the mild OA group, the non-dominant extremity $(\mathrm{P}=0.030)$ between groups of normal OA compared to severe OA, and the non-dominant extremity $(\mathrm{P}=0.021)$ between groups of moderate OA compared to severe OA.

\section{Discussion}

Our study examined changes in kinematics parameters of subjects with knee OA and investigated excursion changes at the hip, knee, and ankle joints during gait in patients with knee OA of varying severity. There was a significant decrease in foot progress angles in the transverse plane in the non-dominant extremity between groups of mild OA and moderate OA.

The external Knee Adduction Moment (KAM) during walking has been shown to be a contributing factor to the advancement of medial compartment of OA knees [19, 20]. The alignment of the lower limb, foot rotation, and foot progression angle affect KAM during walking [21, $22]$. The toe out gait is defined as the increase in external rotation of the foot with regard to the direction of progression. Toe out gait decreases the amount of the second peak of KAM, but does not affect the first peak. The toe in gait is defined as the increase in internal rotation of the foot with regard to the direction of progression. Toe in gait decreases the amount of the first peak of KAM.
The mechanism is not well known about the effect of progression angle to reduce the knee adduction moment. It is hypothesized that toe out gait would cause lateral displacement of the center of pressure, accompanied by a medially-shifted knee joint center. This change could decrease the magnitude of KAM by decreasing lever arm of the ground reaction force on the knee joint. It is believed that toe in gait causes medial displacement of the center of pressure, accompanied by a laterally-shifted knee joint center. The net outcome will decrease the lever arm for the vertical ground reaction force in early stance. Toe in gait cannot cause changes in peak knee flexion moment [23].

Therefore, one of the justifications of increasing foot progression angle in mild group could be a compensatory mechanism to reduce the second peak of the KAM. However, decrease in foot progress angles of the moderate group could be the result of disease progression or adopting different methods of compensation mechanism (toe in gait) to decrease the first peak of the KAM.

There was a significant increase in the range of foot eversion/inversion excursion in the non-dominant extremity between groups of normal and mild OA patients, while there was a significant decline in the non-dominant extremity between groups of mild OA and severe OA patients. Patients with knee OA had an increased late stance KAM and a decreased medial-lateral hamstring activation ratio in comparison with the healthy group. Also, external rotation of foot decreased the late stance KAM, medial-lateral shear force, and medial-lateral hamstring activation ratio.

However, internal rotation of foot did not increase these measures. Changes in the position of the foot during gait can change both external load of knee and hamstring muscle activation patterns. This may unload the knee articular cartilages [24]. Therefore, external rotation occurs at the ankle as a compensatory mechanism. Women with mild OA by increasing lateral hamstring activity and external ankle rotation can reduce KAM magnitudes. This strategy did not help people with severe symptoms [25]. Regarding the reason why there appears an increase in the range of eversion/inversion excursion in patients with mild OA compared with healthy people, it can be said that this event may be a compensatory mechanism to reduce KAM. This may have implication in helping to unload the knee articular cartilage. In addition, recent evidence has shown that ankle joint OA is not uncommon in patients with severe knee OA and malalignment of the extremity [26]. 
To explain the reduction of the range of eversion/inversion excursion in patients with severe OA compared with the mild group, we can attribute it as a mechanism for joint stability, which can justify a decrease in the range of eversion/inversion excursion in patients with severe OA. In a person with reduced or rigid eversion/ inversion, the subtalar joint compensates for this instability. Besides studies show that ACL injury in patients with knee OA and instability would happen. Results support that ACL injury predisposes knees to osteoarthritis [27] as the ACL injuries may cause knee proprioception impairment [28]. Therefore, that could be one of the justifications for decreasing eversion/inversion range of the ankle joint in patients with severe OA, which agrees with our findings.

There was a significant decrease in the range of knee flexion/extension excursion between the dominant and non-dominant extremity in the normal group compared to the severe OA group. There was a significant decrease in the range of knee flexion/extension excursion between the dominant and non-dominant extremity in the mild group compared to the severe OA patients; while there was a significant decrease in the dominant extremity between groups of moderate OA patients compared to severe OA patients.

In six studies on patients with knee OA, knee flexion during gait decreased almost 18\% [6]. Research has shown a reduction in knee extension in early stance and a reduction in knee flexion in swing phase. However, the effect of reduced knee flexion during gait in mild to moderate stages of osteoarthritis is more important. Astephen et al. (2008) showed that decreased knee flexion in the stance phase with osteoarthritis would progress. Thus, according to the results of such studies, physical treatments for people with mild to moderate osteoarthritis must be focused on restoring the lost flexion. Anterior knee pain or muscle weakness may be associated with reduced knee flexion during gait [9].

Maly et al. (2008) showed that pain intensity had a relationship with the dynamic range of flexion-extension during gait. OA individuals had smaller knee flexion/extension excursion and higher pain intensity during gait. Although the relationship between the limited ROM, muscle weakness, and pain in patients with osteoarthritis is unclear, but the rehab program can be focused on muscle strengthening to improve ROM and knee pain relief [33].

The researchers report that the sagittal plane excursion of knee during gait is directly related to the pathology of knee injury. Fewer studies report the impact of these changes on other lower extremity joints. Limited range of flexion in stance phase of gait changes shock absorption stage. Impaired knee extension at the last stage of stance increases energy consumption at all stages of gait and reduce stability. The range of motion of the knee is a distinctive characteristic, and an awareness of the impact of the disorder on the periphery of the lower limb joints may be helpful in advancing rehab programs [29].

There was a significant increase in the range of knee abduction/adduction excursion between the dominant limbs of the normal group compared to the severe OA group; there was a significant increase in the range of knee abduction/adduction excursion between the dominant of the mild OA group compared to the severe OA group.

In the study of biomechanical changes in gait of subjects with medial knee osteoarthritis, they achieved high dynamic peak varus [30]. The patients with knee OA in the early stages had a reduced axial tibial rotation, while patients in the acute stage show increased knee adduction [10], which agrees with our findings.

There was a significant decrease in the range of knee internal/external rotation excursion in the non-dominant extremity between groups of mild OA compared to severe OA.

To explain the reduction in the range of knee internal/ external rotation excursion in patients with severe $\mathrm{OA}$ compared with mild OA, this event may indicate disease progression and the effectiveness on the range of motion in horizontal plane of knee joint in patients with severe OA. Varus alignment and knee laxity is higher in patients with OA. Individuals with osteoarthritis had less knee motion and higher muscle co-activation during weight bearing and single support. Higher muscle co-activation and knee stiffening are risk factors of joint integrity [13]. Another view of decrease the range of motion in the knee can be the cause of surrounding muscle co-contraction. That referred to as a factor in reducing the progression of $\mathrm{OA}$ in the resources.

There was a significant increase in the range of hip internal/external rotation excursion between the dominant and non-dominant extremity in the normal group compared to the moderate OA group. There was a significant increase in the range of hip internal/external rotation excursion between the dominant and non-dominant extremity of the normal group compared to the severe OA group, while there was a significant increase in the dominant extremity between groups of mild OA and severe OA. 
It was suggested that some patients with knee OA adopt compensatory gait strategies that serve to unload the medial compartment of the knee [31]. Studies have reported a reduction in the second (but not the first) peak of KAM in patients with $\mathrm{OA}$ when the foot is externally rotated between $10^{\circ}$ and $21^{\circ}$ beyond the natural foot position during walking $[21,24]$. Indeed, a negative correlation between the amount of the second peak KAM and the toe out angle has been consistently reported in patients with OA and also healthy subjects.

The lack of consensus regarding the reductions in the first peak of the knee adduction moment achieved with a toe out gait could relate to the fact that this foot position can result from external rotation at either the ankle or hip. the mechanism to reduce the first peak KAM (detailed above) requires external rotation of the knee joint axis, which can only be achieved via external rotation at the hip [32]. The increase in the range of hip external rotation excursion could be a compensatory mechanism in patients with knee OA of varying severity. This effect is more pronounced with increasing severity of osteoarthritis.

There was a significant increase in the range of anterior/posterior pelvic tilt excursion between the dominant extremities of the normal group compared to the severe OA group. There was a significant increase in the range of anterior/posterior pelvic tilt excursion between the dominant and non-dominant extremity of the mild OA group compared to the severe OA group. There was a significant increase in the range of anterior/posterior pelvic tilt excursion between the dominant extremities of the moderate group compared to the severe OA group.

Investigations have shown that individuals with osteoarthritis compared with healthy people have more anterior pelvic tilt, pelvic swimming, smaller hip flexor moments and knee abduction and knee extensor during stance. In addition, patients with severe knee OA has shown greater ankle plantar flexion, knee extension, and hip abduction [15].

There was a significant decrease in the range of lateral pelvic tilt excursion in the non-dominant extremity between groups of normal compared to mild OA. There was a significant decrease in the non-dominant extremity between groups of normal OA compared to severe OA. Finally, there was a significant decrease in the nondominant extremity between groups of moderate OA compared to severe OA.

Our study findings will enable physical therapists to develop strategies focused on the mechanical malfunc- tion, improving the individual's performance or even helping to slow the OA progression. Patients in each severity level could use different strategies to reduce pain.

\section{Acknowledgments}

Hamedan Islamic Azad University has financially supported the present paper. The authors would like to express their sincere thanks to all participants for their assistance. The authors gratefully acknowledge support for this work provided by Islamic Azad University, Hamedan Branch, Hamedan, Iran.

\section{Conflict of Interest}

The authors declared no conflicts of interest.

\section{References}

[1] Gorevic PD. Osteoarthritis: A review of musculoskeletal aging and treatment issues in geriatric patients. Geriatrics. 2004; 59(8):28-32. PMID: 15332414

[2] Liikavainio TU. Biomechanics of gait and physical function in patients with knee osteoarthritis: Diagnosis and treatment of knee osteoarthritis. Finland: Publication of the University of Eastern; 2010.

[3] Østerås N, Risberg MA, Kvien TK, Engebretsen L, Nordsletten L, Bruusgaard D, et al. Hand, hip and knee osteoarthritis in a Norwegian population-based study - The MUST protocol. BMC Musculoskeletal Disorders. 2013; 14:201. doi: 10.1186/1471-2474-14-201

[4] Illyés Á, Kiss RM. Gait analysis of patients with osteoarthritis of the hip joint. Facta Universitatis-Series: Physical Education and Sport. 2005; 3(1):1-9.

[5] Stauffer RN, Chao EY, GyÖry AN. Biomechanical gait analysis of the diseased knee joint. Clinical Orthopaedics and Related Research. 1976; 126:246-55. doi: 10.1097/00003086197707000-00048

[6] Ornetti P, Maillefert JF, Laroche D, Morisset C, Dougados M, Gossec L. Gait analysis as a quantifiable outcome measure in hip or knee osteoarthritis: A systematic review. Joint Bone Spine. 2010; 77(5):421-5. doi: 10.1016/j. jbspin.2009.12.009

[7] Kaufman KR, Hughes C, Morrey BF, Morrey M, An K-N Gait characteristics of patients with knee osteoarthritis Journal of Biomechanics. 2001; 34(7):907-15. doi: 10.1016/ s0021-9290(01)00036-7

[8] McGibbon CA, Krebs DE, Scarborough DM. Rehabilitation effects on compensatory gait mechanics in people with arthritis and strength impairment. Arthritis \& Rheumatism 2003; 49(2):248-54. doi: 10.1002/art.11005 
[9] Astephen JL, Deluzio KJ, Caldwell GE, Dunbar MJ. Biomechanical changes at the hip, knee, and ankle joints during gait are associated with knee osteoarthritis severity. Journal of Orthopaedic Research. 2008; 26(3):332-41. doi: 10.1002/jor.20496

[10] Nagano Y, Naito K, Saho Y, Torii S, Ogata T, Nakazawa $\mathrm{K}$, et al. Association between in vivo knee kinematics during gait and the severity of knee osteoarthritis. The Knee. 2012; 19(5):628-32. doi: 10.1016/j.knee.2011.11.002

[11] Al-Zahrani KS, Bakheit AMO. A study of the gait characteristics of patients with chronic osteoarthritis of the knee. Disability and Rehabilitation. 2002; 24(5):275-80. doi: 10.1080/09638280110087098

[12] Childs JD, Sparto PJ, Fitzgerald GK, Bizzini M, Irrgang JJ. Alterations in lower extremity movement and muscle activation patterns in individuals with knee osteoarthritis. Clinical Biomechanics. 2004; 19(1):44-9. doi: 10.1016/j.clinbiomech.2003.08.007

[13] Schmit LC, Rudolph KS. Influences on knee movement strategies during walking in persons with medial knee osteoarthritis. Arthritis \& Rheumatism. 2007; 57(6):1018-26. doi: 10.1002/art.22889

[14] Thorp LE, Sumner DR, Block JA, Moisio KC, Shott S, Wimmer MA. Knee joint loading differs in individuals with mild compared with moderate medial knee osteoarthritis. Arthritis \& Rheumatism. 2006; 54(12):3842-9. doi: 10.1002/art.22247

[15] Huang SC, Wei IP, Chien HL, Wang TM, Liu YH, Chen $\mathrm{HL}$, et al. Effects of severity of degeneration on gait patterns in patients with medial knee osteoarthritis. Medical Engineering \& Physics. 2008; 30(8):997-1003. doi: 10.1016/j.medengphy.2008.02.006

[16] Landry SC, McKean KA, Hubley-Kozey CL, Stanish WD, Deluzio KJ. Knee biomechanics of moderate OA patients measured during gait at a self-selected and fast walking speed. Journal of Biomechanics. 2007; 40(8):1754-61. doi: 10.1016/j.jbiomech.2006.08.010

[17] Taki S, Arshi A, Navvab MF, Yazdi H. Lower extremity kinematic analysis of medial tibiofemoral osteoarthritis patients in frontal plane using principal components analysis (PCA). Modares Mechanical Engineering. 2014; 14(2): 159-166.

[18] Guide VP. Foundation notes revision 2.0 March 2010: For use with Plug-in Gait Version; 2010.

[19] Lynn SK, Reid SM, Costigan PA. The influence of gait pattern on signs of knee osteoarthritis in older adults over a 5-11 year follow-up period: A case study analysis. Knee. 2007; 14(1):22-8. doi: 10.1016/j.knee.2006.09.002

[20] Foroughi N, Smith R, Vanwanseele B. The association of external knee adduction moment with biomechanical variables in osteoarthritis: A systematic review. Knee. 2009; 16(5):303-9. doi: 10.1016/j.knee.2008.12.007

[21] Guo M, Axe MJ, Manal K. The influence of foot progression angle on the knee adduction moment during walking and stair climbing in pain free individuals with knee osteoarthritis. Gait \& Posture. 2007; 26(3):436-41. doi: 10.1016/j.gaitpost.2006.10.008
[22] Teichtahl AJ, Morris ME, Wluka AE, Baker R, Wolfe R, Davis SR, et al. Foot rotation-A potential target to modify the knee adduction moment. Journal of Science and Medicine in Sport. 2006; 9(1-2):67-71. doi: 10.1016/j. jsams.2006.03.011

[23] Shull PB, Shultz R, Silder A, Dragoo JL, Besier TF, Cutkosky MR, et al. Toe-in gait reduces the first peak knee adduction moment in patients with medial compartment knee osteoarthritis. Journal of Biomechanics. 2013; 46(1):122-8. doi: 10.1016/j.jbiomech.2012.10.019

[24] Lynn SK, Costigan PA. Effect of foot rotation on knee kinetics and hamstring activation in older adults with and without signs of knee osteoarthritis. Clinical Biomechanics. 2008; 23(6):779-86. doi: 10.1016/j.clinbiomech.2008.01.012

[25] Motlagh FN, Rostami M, Emrani A, Yazdi H, Keyhani M. Ankle rotation changes and its influences in knee osteoarthritis. Medical Journal of the Islamic Republic of Iran. 2013; 27(2):67-76.

[26] Tallroth K, Harilainen A, Kerttula L, Sayed R. Ankle osteoarthritis is associated with knee osteoarthritis. Conclusions based on mechanical axis radiographs. Archives of Orthopaedic and Trauma Surgery. 2007; 128(6):555-60. doi: $10.1007 /$ s00402-007-0502-9

[27] Ajuied A, Wong F, Smith C, Norris M, Earnshaw P, Back D, et al. Anterior cruciate ligament injury and radiologic progression of knee osteoarthritis. The American Journal of Sports Medicine. 2013; 42(9):2242-52. doi: $10.1177 / 0363546513508376$

[28] Relph N, Herrington L, Tyson S. The effects of ACL injury on knee proprioception: A meta-analysis. Physiotherapy. 2014; 100(3):187-95. doi: 10.1016/j.physio.2013.11.002

[29] Kirkwood RN, Resende RA, Magalhães C, Gomes HA, Mingoti SA, Sampaio RF. Application of principal component analysis on gait kinematics in elderly women with knee osteoarthritis. Brazilian Journal of Physical Therapy. 2011; 15(1):52-8. PMID: 21519716

[30] Silva HGPV da, Cliquet Junior A, Zorzi AR, Miranda JB de. [Biomechanical changes in the gait of individuals with medial knee osteoarthritis (Portuguese)]. Acta Ortopédica Brasileira. 2012; 20(3):150-6. doi: 10.1590/s141378522012000300004

[31] McGibbon CA, Krebs DE. Compensatory gait mechanics in patients with unilateral knee arthritis. The Journal of rheumatology. 2002; 29(11):2410-9. PMID: 12415602

[32] Reeves ND, Bowling FL. Conservative biomechanical strategies for knee osteoarthritis. Nature Reviews Rheumatology. 2011; 7(2):113-22. doi: 10.1038/nrrheum.2010.212

[33] Maly RC, Stein JA, Umezawa Y, Leake B, Anglin MD. Racial/ethnic differences in breast cancer outcomes among older patients: effects of physician communication and patient empowerment. Health Psychology. 2008; 27(6):72836. doi: $10.1037 / 0278-6133.27 .6 .728$. 